\section{Los materiales bio basados y el paradigma desarrollista latinoamericano: perspectivas desde el Diseño industrial}

Sergio Donoso ${ }^{(1)}$ y Andrea Wechsler ${ }^{(2)}$

\begin{abstract}
Resumen: La teoría desarrollista plantea que el progreso de los países se basa en la industrialización y sustitución de importaciones, en contra del desarrollo extractivo, que ha sido el paradigma latinoamericano en las últimas décadas. Los nuevos modos de agregación social y la cultura DIY, proponen una economía circular para el desarrollo sustentable, incluyendo biomateriales elaborados por las propias comunidades. Sin embargo, aquellos no son necesariamente materiales industriales por carecer de homologación, asunto que plantea profundas implicancias al diseño.

Presentamos un estado del arte latinoamericano, acerca de comunidades que producen sus propios materiales y algunos casos donde el diseño ha intervenido.
\end{abstract}

Palabras clave: biomateriales - economía circular - cultura maker - diseño sustentable, desarrollismo

[Resúmenes en inglés y portugués en las páginas 93-94]

(1) $\mathrm{PhD}$. Doctor en Diseño del politécnico de Milan y Diseñador de Productos de la Universidad de Valparaíso, actualmente realizando las guías de los cursos de Seminario de Procesos Productivos en la Carrera de Diseño Industrial. Dentro de su experiencia destaca el desarrollo de redes vinculando el diseño con las PYMES

(2) PhD Built Environment (UNSW), Diseñadora Industrial (UBB), Licenciada en Diseño (UBB). Durante su experiencia profesional se ha desempeñado en desarrollar proyectos de investigación y desarrollo para ser financiados por fondos gubernamentales. Además, se ha desempeñado como diseñadora y docente en cursos de postgrado en Australia.

Ambos pertenecen al Departamento de Diseño, Universidad de Chile. 


\section{Desarrollismo y cultura tecnológica}

\subsection{La teoría desarrollista}

Originada en Latinoamérica en 1949 en la CEPAL, propuso una hoja de ruta para el desarrollo continental, donde se planteó que el progreso de los países se basaba en la industrialización y sustitución de importaciones, pero con la intervención del Estado como agente regulador(Bresser-Pereira, 2017). La economía y la producción en general, adquirió un cierto matiz nacionalista, considerado entonces como algo válido, pues se estimaba que la competencia por la producción era entre naciones y no entre mercados. En este modelo, el interés del Estado era el desarrollo económico, la acumulación del capital y el progreso tecnológico, y no en el lucro empresarial. De esta manera se consideraba que el mercado no podía autorregularse solo según la oferta y la demanda, pues en medio estaba comprometido el progreso de la sociedad y era misión del Estado velar por ello.

El desarrollismo dejó un imaginario nostálgico en la cultura tecnológica que cada cierto tiempo reaparece, en un reclamo al regreso de la producción industrial y donde el Diseño tuvo un destacado protagonismo de la mano del Estado (Bonsiepe, 2016). En efecto, el Diseño llega a Chile como un instrumento de la política pública para garantizar el acceso masivo a los bienes producidos por la industria (Castillo, 2010).Sin embargo, el modelo de "desarrollo hacia adentro" no pudo lograr sus propósitos, debido a que el tamaño del mercado interno(Pino, 2003) no fue el suficiente para soportar un aumento de una producción que lo hiciera sustentable y la constante subvención del Estado comprometía la inversión social en otros ámbitos.

El desarrollismo nacionalista, prevalente en Latinoamérica, buscaba la independencia tecnológica y se contraponía al desarrollo basado en lo extractivo ${ }^{1}$, sin embargo, su término abrupto debido a la dictadura militar dejó muchas dudas acerca de su eficiencia final, pues la rentabilidad debe medirse en el tiempo. El neoliberalismo y la apertura a los mercados llevó aparejado un aumento en el consumo de bienes importados en los cuales no se había alcanzado un alto nivel de desarrollo y produjo una modernización tecnológica en el país.A pesar de este éxito inicial, el neoliberalismo al final ha supuesto un bajo desarrollo económico, baja estabilidad financiera y un aumento de la inequidad (Bresser-Pereira, 2017), que tienen en entredicho su vigencia y que ha generado movimientos reaccionarios por todo el mundo.

A pesar de todo, el desarrollismo aún presente en Latinoamérica, ha sido amenazado por el boom extractivo de materias primas requeridas por las grandes economías, que ha resultado en el ingreso de grandes cantidades de divisas que han afectado el tipo de cambio y cuyo efecto principal es la depresión de la industria manufacturera, conocida como "Enfermedad holandesa" (Campos \& López, 2017) y de la cual padece la economía chilena en la actualidad. Sin embargo, economías como la argentina y la brasileña, que aún mantienen activos algunos elementos del desarrollismo vía aranceles, no se han visto mayormente afectadas pues han diversificado su matriz productiva y no son dependientes de los recursos naturales, al nivel chileno. 
Hablar entonces del desarrollismo sólo desde un punto de vista económico, es inoficioso pues desconoce las biografías de las distintas comunidades y sus imaginarios(Rojas, 2006), e impone un modo único de progreso, donde se afirma que la sustitución de importaciones a través de la producción, parece ser el mejor camino para lograrlo.

En Chile ha habido varios intentos de industrialización; a fines del siglo XIX (Pino, 2003), durante las décadas de 1940 y luego durante los años 1960, sin embargo, ninguna de ellas logró la industrialización del país pues nunca consolidaron la cultura tecnológica como una clave de lectura de la sociedad hacia la transformación (Pino, 2003). Más aún, se consolidó y validó el modelo extractivo como la fuente de riqueza, produciendo el lento declive de la industria chilena.

En la actualidad la evolución del modelo desarrollista empieza a tener claras distinciones con el modelo teórico neoliberal, pues se cruza con un nuevo eje; la dignidad. La evolución se dirige hacia la promoción de la política activa para reducir las desigualdades (Bresser-Pereira, 2017). Esto ha calado hondo en las nuevas estructuras sociales que han surgido en las últimas décadas donde distintas comunidades han cuestionado el modelo extractivo, por ser poco sustentable y el concepto de sustitución ya no se refiere solamente por importaciones, sino que se ha ampliado y se refiere también todo aquello que garantice independencia, incluyendo las materias primas y la energía. Estos movimientos tienen el potencial de ser unos catalizadores más permanentes de un nuevo proceso de industrialización pues están acompañados de un cambio profundo en la cultura. Los países más desarrollados han transitado de un paradigma desarrollista a otro, como resultado de su evolución histórica y de las capacidades que han alcanzado y, en aquellos lugares donde no ha existido un tránsito armónico entre ambos, se ha debido a que los catalizadores han sido externos y cuando su influencia ha desaparecido, el proceso se ha detenido.

\subsection{La cultura tecnológica chilena}

La industrialización acelerada ha sido desde mediados del siglo XX uno de los paradigmas para el desarrollo de los países. Sin embargo, la sola disponibilidad de tecnologías para transformar la materia no basta para consolidar un proceso de industrialización. En efecto, uno de los elementos cruciales para su consolidación es la presencia de una cultura tecnológica fuerte de un determinado grupo humano, que distingue sus operaciones de las de otros y agrega un sello de valor. En este sentido, hubo esfuerzos sostenidos por consolidar la educación tecnológica, incluso recurriendo a reformas de modelos educativos (Donoso \& Vielma, 2019), pues la industrialización está aparejada de especialización y aprendizaje crítico.

La naturaleza de la cultura tecnológica no es homogénea, pues existen otros factores gravitantes que afectan la industrialización y son los acuerdos morales o "valores". Estos, tienen raíces muy profundas y antiguas que condicionan las formas de producir o transformar. Sin ir más lejos, la identidad chilena (Larraín, 2001) aporta un punto de vista sobre la producción pues está estrechamente ligada a los procesos modernizadores. Los valores pueden tener un centro más bien tradicionalista o secular (Kistler, Thöni, \& Welzel, 2015); $\mathrm{o}$ ir desde la subsistencia a la autorrealización. Estas consideraciones son de tal manera 
influyentes, que dos culturas con sistemas valóricos muy distintos, puede operar la misma tecnología de maneras muy distintas y en consecuencia no es posible obtener resultados idénticos a todo evento (Donoso, Investigación cualitativa en Diseño y Artes, 2019).

Los distintos paradigmas productivos pueden ser más o menos aceptados según afecten o no los modos culturales, es así como existen países con un determinado nivel de desarrollo("Objetivos de desarrollo sostenible," 2019), pero con una distribución interna más homogénea en tanto que otros, como Chile, presentan profundas diferencias que al final afectan la producción y los modos de producir.

Hablábamos de los paradigmas productivos, pues si hacemos un recorrido por la historia, veremos que los ejes del poder han cambiado en el tiempo; desde una sociedad agraria a una industrial y luego a otra basada en el conocimiento o era postindustrial (Cohen, 2007), para llegar ahora a la llamada industria 4.0 (Schwab, 2016). En el primer paradigma, el eje del poder estaba del lado de quien controlaba un territorio y fue la época de las conquistas, en el segundo paradigma, el poder estaba del lado del capital y ya no era necesario conquistar territorios, bastaba comprar materia prima. El tercer paradigma fue más radical aún, pues el poder estaba del lado de quienes producían conocimientos, entonces territorio y capital pasaron a segundo plano pues el poder ahora es global y virtual, rodeado por la noosfera(Wilson, 2017). Sin embargo, ¿Qué sucede en Chile donde conviven los tres paradigmas? ¿Es un freno al ingreso a la cuarta revolución industrial?

Los procesos de industrialización requieren que la comunidad internalice los procesos de cambios como parte de la evolución cultural de la sociedad misma y no participe solo como actores-observadores del cambio. La identidad nace entonces desde una posición diferenciada y activa, adoptada frente otros países, mediante una realidad construida (Berger \& Luckmann, 2014) socialmente a través de acuerdos participativos que han cohesionado a la comunidad. Desde lo tecnológico, implica tanto una diferenciación como una independencia que mejora la posición competitiva del país y que entre otros se verifica en la cantidad de patentes otorgadas.

El desarrollismo y su visión tecnocrática, muchas veces ignorante de la cultura tecnológica de las distintas comunidades y los afectos que los vinculan, conllevó grandes distorsiones en distintos lugares de Chile al desconocer, por ejemplo, aspectos tan sutiles como los mitos fundacionales o sobrenaturales (Eliade, 2013). Estas construcciones humanas afectan de manera decisiva la postura social frente al trabajo y en consecuencia a la ética del trabajo (Weber, 2011). Este aspecto, no menor, ha visto sus peores resultados en los fracasados procesos de reconversión laboral de fines del siglo XX, donde se pensaba que un trabajador podría cambiar de un tipo de manufactura a otra, sólo con capacitación laboral (Aravena \& Betancur, 1999); es así comose ha visto a mineros tejiendo suéteres o elaborando pastelería. El tiempo ha demostrado que casi todas las iniciativas centralizadas, propias del desarrollismo, o "Top Down", tienden al fracaso y las que más resultan exitosas son aquellas "bottom up", es decir las que se originan desde la base comunitaria, pues poseen el soporte cultural que las hace sustentables en lo social.

En la medida que Chile se ha desarrollado en lo económico, la cultura tecnológica ha cambiado, pero sobre todo lo ha hecho el sistema de valores, que se ha ido desplazando cada vez más hacia la autorrealización (Kistler et al., 2015). Este fenómeno se produce cuando 
un país supera los niveles de pobreza y sus habitantes empiezan a escalar en la pirámide de las necesidades. En este momento, Chile atraviesa este proceso y la población ha empezado a preocuparse de otros aspectos de la vida, que tienen que ver con la autorrealización y los temas que hace sólo una década eran irrelevantes, ahora resultan gravitantes, tal como ocurre con la sustentabilidad.

\subsection{La extensión del concepto de sustentabilidad}

Es sabida la implicancia y extensión del concepto de sustentabilidad, sin embargo, desde las visiones más estructuralistas, ha decantado hacia una comprensión más centrada en los efectos en lo humano que tienen sus distintas dimensiones:

Sustentabilidad económica: se fomenta el desarrollo económico, mediante la maximización de la productividad,pero sin afectar negativamente lo social y medioambiental, pues si ello ocurre, se degrada la habitabilidad. Es decir, decrece el índice de desarrollo humano y se debilita el volumen del capital social. Por otra parte, se busca minimizar la importación de insumos y la exportación de residuos, efectos colaterales degradantes o cualquier otro tipo de externalidad negativa (Fernández et al., 1999). Sin embargo, se requiere establecer marcos regulatorios a las condiciones de competitividad para no afectar el entorno, como, por ejemplo, recibir sólo algunos tipos de empresas. En cualquier caso, la sustentabilidad económica debe medirse en el largo plazo.

Sustentabilidad social: en cuyo eje está el desarrollo humano, propone la mejora de toda la sociedad para superar los umbrales de pobreza, mediante el potenciamiento de las redes de auto organización ciudadana y el fomento de los micro proyectos de desarrollo. La manera de lograrlo es a través de la consolidación de la economía popular para interactuar con lo público y lo privado. Para ello se hace necesario regular los procesos de movilización demográfica (Fernández et al., 1999); no cerrando fronteras, sino que generando políticas macro territoriales en búsqueda de la integración. El concepto de ciudad creativa nace en territorios permeables al intercambio de ideas y por ello el flujo de migrantes es uno de activos de la nueva economía (Florida, 2010); todos los países desarrollados son multiculturales.

Sustentabilidad ecológica: la visión ecológica ha evolucionado y reconoce a la tecnología como un capital gravitante en el desarrollo y no solo como un actor depredador. De esta manera se busca enriquecer el capital natural mediante el capital tecnológico, como un proceso de agregación de valor. El capital tecnológico debe apuntar a la autosuficiencia, lo mismo que en lo económico, tendiendo a reducir la importación de materias y energías y también reducir la exportación de residuos. Se considera que la tecnología es un aporte cuando no hay o es mínimo el impacto ambiental y en el nuevo desarrollismo se favorece la no dependencia extractiva. La sustentabilidad ecológica requiere reconocer los capitales sociales, culturales, tecnológicos y naturales de la comunidad a fin de ayudar a consolidar 
territorios sustentables, que eventualmente se consoliden como distritos sustentables, medidos también en el consumo de recursos naturales, utilizados en lo urbano.

Este conjunto de dimensiones finalmente afecta la gobernabilidad (Figura 1) que es lo que apoya la sustentabilidad política. A su vez la gobernabilidad busca reducir la presión de los procesos productivos globales en favor de lo local para reducir la exclusión social (Fernández et al., 1999). El fortalecimiento de la gobernanza local y la rearticulación de las organizaciones micro sociales ayuda a enfrentar las decisiones extra locales. Por otra parte, se ha visto recientemente que el neoliberalismo ha dejado claro que el éxito macroeconómico no puede sustentarse en un fracaso microeconómico pues degrada la sustentabilidad política.

Descentralizar y establecer mecanismos democráticos de participación ciudadana para llevar adelante acciones participativas permite elaborar planes estratégicos representativos y agendas locales significativas. Donde la política de desarrollo de las comunidades es distinta para cada una de ellas porque debe considerar sus entornos y contextos regionales, pues la sustentabilidad opera sobre un territorio específico.

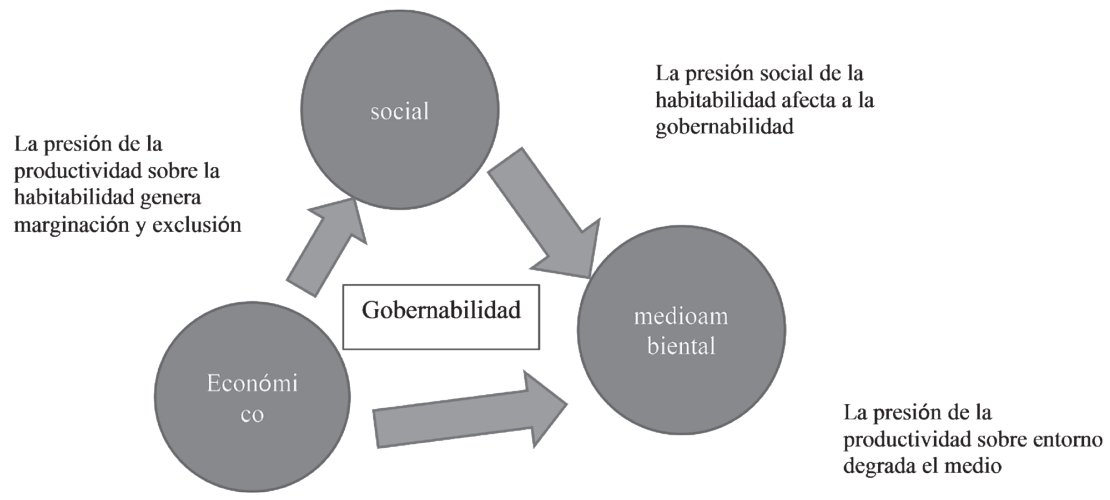

Figura 1

\subsection{La economía circular}

En función de los serios problemas medioambientales que se están generando producto de nuestro sistema de consumo actual, el Programa Medioambiental de las Naciones Unidas (UNEP) desarrolló un marco de política general llamada "Consumo y Producción Sustentable" (SCP) (UNEP). Este marco ha sido conformado por enfoques a la producción y consumo sustentables tales como la ecología industrial, la que se ha definido como 
la "ciencia del desarrollo sustentable" (Graedel \& Allenby, 1995). La ecología industrial estudia el flujo de energía y materiales a través de los sistemas industriales durante todo el ciclo de vida de un producto con una preocupación particular acerca de los impactos que las actividades industriales tienen sobre el medioambiente (Garner \& Keoleian, 1995). La ecología industrial recomienda en primera instancia especificar materiales reciclados. El principio involucrado es efectivamente transformar la basura en comida de otros procesos (McDonough \& Braungart, 2002).

Chile es un país que cada día busca fortalecer sus políticas medioambientales. Sin embargo, hasta la fecha, solo el 14\% de los residuos son reciclados en Chile (MMA, 2016). En respuesta a este problema, el 17 de mayo del año 2017 se promulgó la ley REP. Esta Ley otorga la responsabilidad del ciclo de vida del producto o servicio al productor. Enmarcada en esta nueva ley, surge un nuevo problema para los industriales, en el que se verán "obligados" a aplicar criterios de la ecología industrial (Graedel \& Allenby) a sus procesos y desarrollo de ciertos productos. La ley REP exige a los productores e importadores a hacerse cargo del fin de vida de sus productos mediante actividades tales como la reutilización de sus propios residuos productivos en sus propios procesos o en la creación de nuevos productos o insumos. La naturaleza obligatoria de la ley REP provocará un desarrollo expansivo de la industria de la valorización de residuos, generando más alternativas de reciclado y nuevos mercados para la inserción de estos productos reciclados, gatillando nuevas cadenas de valor.

\section{Las comunidades autosustentables}

\subsection{Las nuevas comunidades como reacción al neoliberalismo}

Como reacción a los procesos globales de cambio, ha surgido una nueva conciencia y nuevos modos de agregación social; entre ellas, innumerables comunidades que buscan la auto sustentabilidad, tanto en el abastecimiento como en la producción y el intercambio. Este proceso es en esencia urbano (Florida, 2010) e involucra a personas con un alto grado de creatividad y sensibilidad hacia el consumo crítico y el comercio justo. Algunas de ellas son las comunidades conocidas como DIY, sigla que traducida del inglés significa "hágalo usted mismo", que entre sus prácticas comunitarias incluyen la economía circular. Sin ir más lejos, ha surgido una nueva categoría, originada desde el concepto de bolsas de residuos, que buscan que su desarrollo produzca el mínimo impacto sobre el ecosistema y donde sus residuos puedan reconvertirse la mayor cantidad de veces posible en otros bienes o incluso en otros materiales.

Muchas de estas comunidades han nacido al alero de las universidades y luego se han integrado a comunidades productivas colaborativas, como los "open labs" o los "fab labs", donde alguna de ellas ha explorado la idea de reconvertir residuos en otros productos u otros insumos para procesos industriales, en cualquier caso, explorando la innovación disruptiva (Schwab, 2016). Existe una caracterización de parte del Estado de Chile, acerca de la naturaleza de ellas y del potencial para constituirse en un sector económico activo 
(Mapeo de las industrias creativas en Chile. caracterización y dimensionamiento, 2014), muy afín con el concepto de la industria 4.0 y su enfoque en la reducción de la inequidad (Schwab, 2016).

La conciencia ecológica fue el primer aspecto de la sustentabilidad que fue aceptado por la comunidad y luego han seguido la sustentabilidad social y recientemente la económica. Esta última como una de las grandes demandas del reciente movimiento social. El nuevo desarrollismo, escapa del paradigma proteccionista para decantar en una posición de competencia sustentable en vías a una integración internacional, más que a la subordinación (Bresser-Pereira, 2017). Esto soporta los movimientos antiglobalización y da lugar a que las pequeñas comunidades puedan participar del desarrollo de una manera más equitativa y activa, sin el autoritarismo del desarrollismo original.

Esta economía, basada muy a menudo en las ideas y la imaginación (Gilmore \& Pine, 2011), se sustenta también en comunidades virtuales unidas por estilos de vida comunes y extraterritoriales (Powazek, 2002). Entre los resultados, ha estado el nacimiento de la economía creativa o economía naranja (Buitrago \& Duque, 2013), donde el capital cultural ha sido reemplazado por el capital creativo (Florida, 2010) y ha construido un nuevo espacio productivo, basado en el talento (Mapeo de las industrias creativas en Chile. caracterización y dimensionamiento, 2014). Una de las principales características de estas comunidades es el gran desarrollo de los servicios, porque son intensivas en el uso y generación de ellos.

A pesar de todo, las comunidades creativas también presentan externalidades negativas, una de las cuales es la gentrificación, que implica entre otros, el desplazamiento de los antiguos habitantes a los territorios hacia otros más acordes con sus sistemas de valores. Dicho de otra manera, la reacción hacia el neoliberalismo en la búsqueda de autarquía puede generar aislacionismo; justo lo opuesto al intercambio cultural reclamado por la sustentabilidad social, que paradojalmente es un fenómeno resultante del neoliberalismo que facilita la inversión concentrada (López, 2013), a través de subsidios a la clase media para la adquisición de viviendas en zonas de renovación urbana. En Santiago de Chile, esto ha ocurrido en antiguos barrios de reconversión, como los barrios Italia o Bellavista en Santiago, que se ha consolidado como un atractor de comunidades creativas pero que han afectado la matriz cultural del lugar, produciendo la migración de los antiguos habitantes, estimulado también por el aumento del precio del suelo, debido a la presión inmobiliaria (López, 2013).

\subsection{Independencia energética y elaboración de materiales bio basados}

La auto dependencia, uno de los pilares de la sustentabilidad económica y que afecta a la productividad, es uno de los valores compartidos por nuevas comunidades preocupadas por el menor impacto posible sobre el medioambiente. De esta manera surgen grupos auto organizados como comunidades creativas (Meroni, 2007), conscientes del progreso y del crecimiento económico, pero no a costa de la degradación de la habitabilidad o lo que es lo mismo, sobre la calidad de vida. 
Existen diversas iniciativas que apoyan proyectos de desarrollo social y formación de comunidades sustentables que se basan en el manejo de residuos y reciclaje, varias de ellas son fundaciones o empresas B, tales como Recyclápolis, Kyklos o Triciclos que promueven el desarrollo de una industria asociada.

\subsection{Tipos de materiales biobasados, desarrollados por comunidades}

Bajo el alero de este movimiento que promueve la reutilización de residuos, considerando el ciclo de vida completo de los productos, ha dado origen a una serie de iniciativas desde las universidades y centros tecnológicos para posteriormente migrar a las comunidades DIY o a pequeños emprendimientos. Los materiales DIY son materiales de fabricación casera que pueden ofrecer nuevas funciones, expresiones y cualidades estéticas, muchas veces debido a su forma de producción artesanal y al origen de la materia prima (Cores Irago, 2018). En la mayoría de los casos, aquellos no son necesariamente materiales industriales por carecer de homologación, asunto que plantea profundas implicancias al Diseño. Otro problema importante para la aceptación de estos nuevos materiales es que, la mayoría de las veces se han generado como materiales de reemplazo, compatibles con los existentes procesos de producción y por lo tanto deben ser más de igual o menor precio y de mejor calidad que los materiales tradicionales para tomar cuota de mercado (Cores Irago, 2018). Este es un gran desafío ya que por lo general estos materiales bio basados tienen un desempeño mecánico un poco menor a los materiales tradicionales.

En cuanto al sistema de emprendimiento con estos nuevos materiales biobasados, para la comunidad la estrategia principal es la diferenciación, estableciéndose una posible ruta de los modelos de negocio que serían coherentes con los objetivos y la naturaleza del proyecto. Las plataformas de difusión y contacto permiten visibilizar los materiales y buscar colaboración para seguir desarrollando el material y sus posibles aplicaciones. Existen diversos tipos de plataformas de difusión de materiales, estas pueden ser de código abierto o con un perfil más comercial.

La Figura 2 presenta un diagrama del tipo de plataformas de difusión y espacios de creación de nuevos materiales biobasados. 


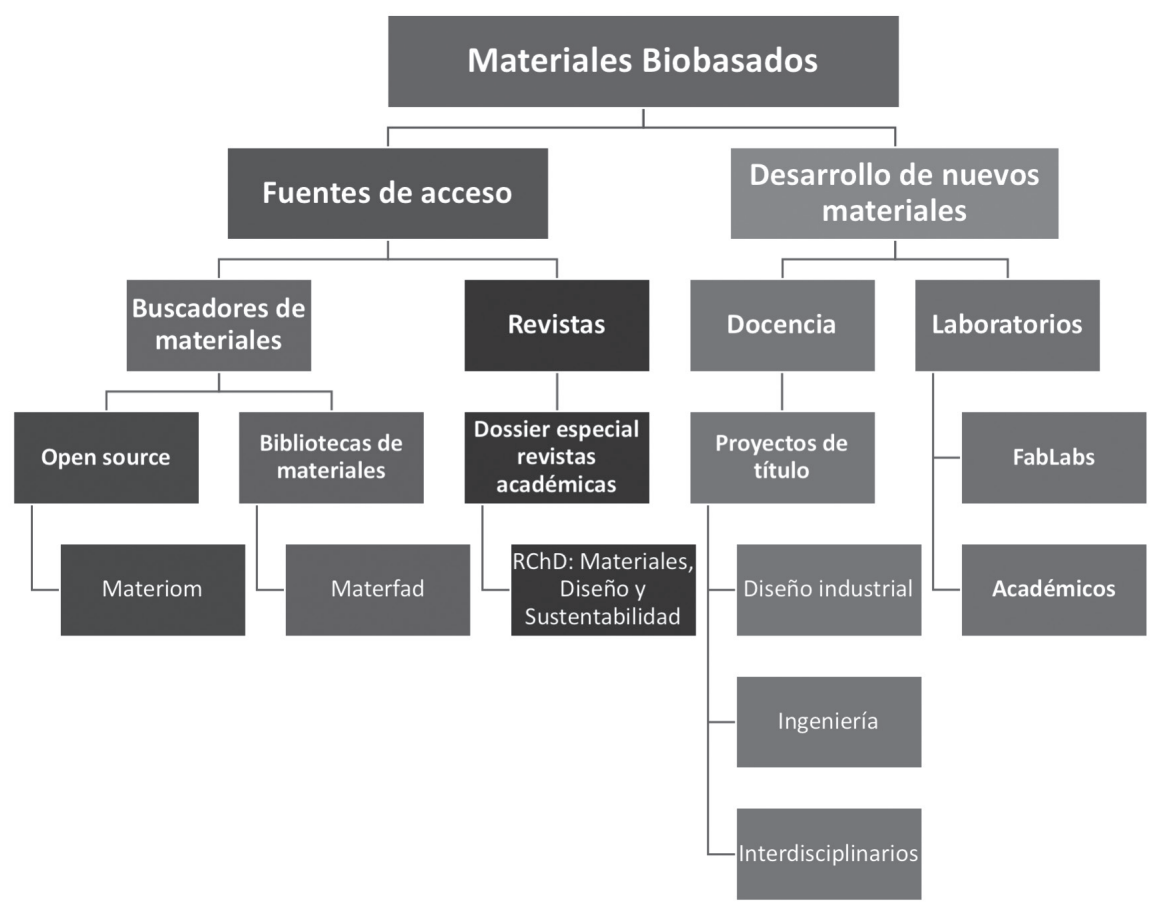

Figura 2. Materiales biobasados

Las plataformas de código abierto presentan una variedad de productos en la que se genera una publicaciónde fórmulas o "recetas" 2 de materiales. Las plataformas pueden ser virtuales, presenciales, como los Fab labs o eventos de difusión, entre otras (Cores Irago, 2018). Algunas de estas plataformas existentes en Chile son:

Materiom: biblioteca digital de código abierto de materiales biobasados. Esta biblioteca entrega "recetas" e información sobre materiales manufacturados con componentes de diversas fuentes naturales; como por ejemplo residuos de la agricultura. Esta plataforma se aprecia en la Figura 3.a. El objetivo de Materiom es el de acelerar el desarrollo de materiales alrededor del mundo ("Materiom: Home," 2019).

FabLab U de Chile: laboratorio de Fabricación Digital ubicado en la Facultad de Ciencias Físicas y Matemáticas de la Universidad de Chile (FCFM). En el FabLab se desarrollan materiales, procesos, prototipos, productos, equipamiento y experiencias de libre acceso que mejoren la calidad de vida de las personas; trabajando en equipos multidisciplinarios de diseñadores, ingenieros y de otras especialidades. Cabe destacar algunos desarrollos 
tales como un neutralizador de aromas y un contenedor de desechos orgánicos con borra de café aglomerada con agar agar ${ }^{3}$, generados por Inostroza y Barros. Otro proyecto de interés fue la modificación de una impresora 3D open source, en la cual se imprimió arcilla, greda y otros materiales biobasados de manera exitosa ("Fablab U.de Chile," 2019).

Laboratorio de Biofabricación UC: alojado en la Facultad de Arquitectura, Diseño y Estudios Urbanos de la Pontificia Universidad Católica de Chile, es un espacio multidisciplinario, en el cual se desarrollan nuevos materiales basados en organismos vivos y sus procesos de biofabricación. Los proyectos ahí realizados se caracterizan por ser tecnologías de bajo costo y libre acceso ("Laboratorio de Biofabricación UC," 2019).

Una de las fortalezas de estas plataformas, es que, aprovechando los materiales y los métodos de obtención publicados en fuentes de código abierto, estos materiales pueden ser replicado o perfeccionados para ser prototipados y caracterizados. Posteriormente podrían ser manufacturados a mayor escala y comercializados por pequeños emprendedores a nivel local.

Existen además plataformas de difusión de materiales con fines más comerciales. En Latinoamérica encontramos a:

Materfad (2019): con sedes en España, México, Colombia y Chile, es un buscador de materiales que presenta un abanico de materiales biobasados. Esta plataforma permite contactar al proveedor o fabricante de los materiales disponibles en el mercado. Materfad Valparaíso (2019), es además el Centro de materiales de la Universidad de Valparaíso, en el cual se desarrolla una labor de orientación, investigación y vigilancia tecnológica en el campo de los nuevos materiales. En esta biblioteca de materiales se pueden encontrar todas las familias de materiales; aún cuando permite filtrar la búsqueda por estrategias de ecodiseño, normativas y ecoetiquetas. Este buscador se muestra en la Figura 3.b.

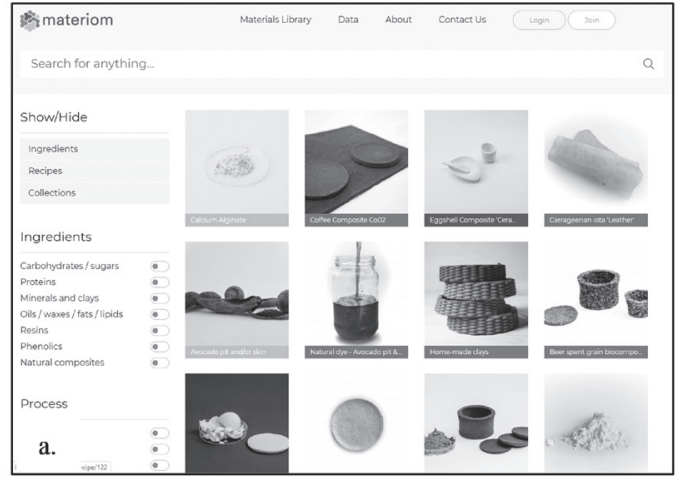

Figura 3.a

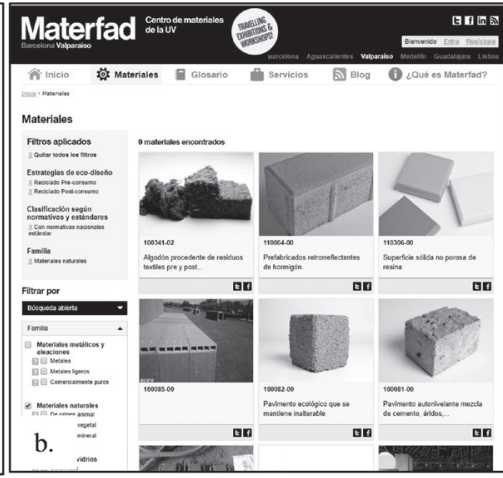

Figura 3.b. 
Además de estas bibliotecas de materiales, se puede mencionar en Chile, otras fuentes de difusión, esta vez una revista académica. Si bien no se han identificado publicaciones periódicas sobre materiales y diseño en Latinoamérica, se han encontrado números de revistas tales como el Dossier de la Revista Chilena de Diseño: Materiales, Diseño y Sustentabilidad, número dedicado exclusivamente a poner en valor el desarrollo de materiales para el diseño desde un prisma de la sustentabilidad (Wechsler, 2018).

\section{Tipos de materiales bio basados identificadosen Latinoamérica}

En la búsqueda no sólo de independencia tecnológica, o la sustitución de importaciones, sino también en que el desarrollo industrial sea sostenible, se han desarrollado diferentes tipos de materiales biobasados llevados adelante principalmente por comunidades académicas e industriales en Latinoamérica. En la mayoría de estos casos se ha optado por esta vía, en contra de la opción extractivista, pues se busca valorizar el capital natural y como resultado inicial se ha llegado a producir tableros de partículas, con sus principales propiedades caracterizadas.

Se consideran como sustentables aquellos materiales basados en residuos con un alto componente de material celulósico vegetal, que cumplan con los siguientes criterios de la ecología industrial: usar insumos locales, usar residuos como recursos, mantener la biodiversidad e incrementar la eficiencia energética (Ayres, 1989; Frosch \& Gallopoulos, 1989; Garner \& Keoleian, 1995; Graham, 2003; Grann, 1997; McDonough \& Braungart, 2002; Robèrt, 1992).

De los procesos extractivos de materia prima, los materiales celulósicos de fibras de las plantas son la forma más abundante de biomasa desechada. Los materiales celulósicos se dividen en 4 grupos, dependiendo de su origen: hojas, tallos, fruta y semilla. Normalmente se utilizan en materiales compuestos las hojas y el tallo debido a sus fibras más largas que le otorgan mayor resistencia mecánica y térmica, con relación al fruto.

El volumen de residuos sólidos generados sólo por la agroindustria puede llegar a 1,5 millones de toneladas al año. Por un lado, estos residuos son altamente contaminantes y generan impactos medio ambientales tales como plagas, gases emanados por la quema y altos costos de traslados a vertederos (ODEPA, 2012; "Plagas en bodegas Promotores de enfermedades," 2014; Parodi Miranda, 2016). Paralelamente, la recolección de estos residuos exige a las compañías agroindustriales disponer espacios de acopio que entorpecen el desplazamiento al interior de ellas.

Entre los materiales biobasados disponibles en Latinoamérica, se destacan aquellos donde las fibras provienen del tallo y otros que provienen de las frutas:

\section{Tallo: Caña de azúcar (Saccharum officinarum:)}

Industrialmente, la empresa Tablopan en Venezuela, fabricó tableros de partícula con el bagazo de la caña de azúcar por más de 40 años, sin embargo, esta planta terminó con su producción por falta de acceso al bagazo de la caña de azúcar (El Palmar, 2009). En cuanto a desarrollo de nuevos materiales basados en el bagazo de la caña de azúcar en La- 
tinoamérica, la Universidad de Guadalajara en México desarrolló un material compuesto con partículas de bagazo de caña de azúcar aglomerado con polietileno de alta densidad (HDPE) reciclado de botellas de leche (Fuentes T., Silva Guzman, Richter, Sanjuan Duenas, \& Ramos Quirarte, 2007; Fuentes Tavalera, Silva Guzman, Sanjuan Duenas, \& Ramos Quirarte, 2005). La caña de azúcar es plantada en más de 110 países a nivel mundial y sus residuos carecen de un proceso de agregación de valor significativo.

\section{Agave Azul (Agave tequilana):}

Investigadores de la Universidad de Guadalajara en México desarrollaron tableros de partículas de densidad media y alta, obteniendo propiedades físico mecánicas comparables con tableros de partículas convencionales (Ramirez-Calderon, Caulfield, Rowell, Iniguez Covarrubias, \& Toriz Gonzales, 2005) (Iñiguez-Covarrubias et al., 2001). Ramírez-Calderón et al. (2005) generaron un compuesto basado en bagazo de agave con polipropileno mediante un proceso de extrusión y mezcla termoquinética.

El agave azul es utilizado para la producción de Tequila en México, y uno de sus principales residuos es el bagazo, que puede llegar a ser el $40 \%$ del peso total la planta. Se utiliza principalmente como combustible y está disponible durante todo el año en las zonas de producción de tequila en México (Iñiguez-Covarrubias, Lange, \& Rowell, 2001; Valenzuela, 2011).

\section{Fruta}

El uso de los residuos de la industria frutícola es una de las áreas menos exploradas en el desarrollo de materiales compuestos. Algunos de los materiales identificados se presentan a continuación:

\section{Carozos de durazno:}

Molina (2016),en su proyecto de título de Diseño Industrial en la Universidad de Chile, generó un material de carozo de durazno aglomerado con polipropileno, obteniendo un incremento en propiedades físicas y una disminución en las propiedades mecánicas al compararlo con su símil madera plástico. Ulloa (2013), en el marco de su tesis de Ingeniero Constructor de la Universidad de Talca, reemplazó la arena y gravilla del concreto por carozos de durazno en distintas proporciones. El compuesto resultante fue más liviano y con una mayor absorción de agua comparado con el concreto tradicional. Chile es uno de los 10 mayores productores mundiales de durazno, generando hasta $35.000 \mathrm{~m} 3$ de carozos al año (FAO, 2017; iQonsulting, 2016). De estos, un $42 \%$ es enviado a vertederos (Idea Consultora, 2012), con un costo de hasta CLP $\$ 43.200 .000$ por temporada por una sola empresa. Del 58\% restante, una parte es donada como biomasa y una gran cantidad es almacenada en los patios de las industrias, atrayendo vectores, tales como roedores, palomas, e insectos; generando problemas de espacio y malos olores (Molina Mora, 2016).

\section{Coco (Cocos nucifera):}

En México, varios investigadores han trabajado en aplicaciones para las fibras del fruto del coco desde los años 50. Por ejemplo, en la Universidad del Norte y la de Colima en Guadalajara, se ha utilizado la estopa de coco aglomerada con plástico reciclado de alta densidad 
para su uso en viviendas (Navarro, 2005; Quintero \& Gonzalez, 2006); y en la Universidad de Guadalajara, Fuentes et al, (1997) se fabricaron tableros de partículas con la madera del tallo del coco, obteniendo propiedades físicas y mecánicas aceptables comparados con tableros de partículas de madera.

\section{Cáscaras de Maní (Arachis hypogaea):}

En Argentina, el Centro Experimental de Vivienda Económica (Conicet, 2007) está desarrollando tableros basados en cáscaras de maní, aglomerada con poliéster, polietileno tereftalato de posconsumo (PET) y cemento, para techos y paredes de vivienda social. Estos tableros han demostrado ser de bajo costo, con buenas propiedades de aislación acústica y livianos (Beretta \& Gatani, 2007).

El principal productor de maní es India con una producción anual al año 2012 de 400,000 ton, seguida por la producción argentina de 300.00 ton (FAO, 2012). Las cáscaras consisten en el 30\% del peso total del maní. La eliminación de estos residuos es un problema para los productores, ya que por sobre los impactos previamente mencionados de la eliminación de residuos de la agricultura, las cáscaras de maní contienen altos contenidos de lignina y bajos contenidos de nitrógeno, lo que la hacen muy difícil de degradar (Beretta \& Gatani, 2007).

\subsection{Usos de materiales bio basados en el Diseño Industrial}

Proponer materiales para la manufactura de un objeto diseñado, es parte las competencias de la disciplina, por ello los criterios de selección de materiales sustentables están dentro de los conocimientos acerca de la ecología industrial.

Según Karana (2014), existe una tendencia hacia soluciones biobasadas en diseño, estimulado por la visión de un mundo limpio y ecológicamente amigable, donde la producción debiese estar basada en materias primas renovables y los productos debiesen ser reciclables y biodegradables.

Existen diversos enfoquespara desarrollar un material biobasado, que puede ir, por ejemplo, desde la ingeniería en materiales o el Diseño industrial, hasta el trabajo independiente de pequeños emprendedores. Día a día el Diseño se ha involucradocada vez más en el desarrollo de materiales, ya sea por investigaciones formales en trabajos de titulación de pre y posgrado, asignaturas de la carrera de Diseño industrial, concursos y trabajo interdisciplinario y desarrollos generados bajo el movimiento DIY.

El estudio de materiales y procesos es común en el currículo académico de todas las carreras de pregrado de Diseño Industrial y este aspecto ha influido en un fortalecimiento de la cultura tecnológica, que se ha evidenciado en la implementación de laboratorios al interior de ellas.

En Chile existen diversas experiencias en el desarrollo de materiales biobasados y sus aplicaciones, entre ellos destacan: 


\section{Universidad del Biobío}

El Laboratorio de Materiales Compuestos en la Universidad del Biobío en Chile se ha especializado en el análisis, ensayos y desarrollo tecnológico para la producción de tableros de partículas y adhesivos. Varios estudiantes de pregrado de la carrera de Diseño Industrial han llevado a cabo sus proyectos de titulación en este Laboratorio. Estos incluyen tableros para uso interior manufacturados con coligüe (Chusquea culeou), un tipo de bambú chileno; equipos modulares de materiales compuestos madera plástico de polvo de madera de pino y polipropileno extruido y prensado (Wechsler, 2004). Después de estas experiencias, el Laboratorio ha continuado trabajando con diseñadores industriales, incluyendo proyectos de investigación sobre el desarrollo de marcos de ventanas y puertas de compuestos madera plástico.

\section{LincUdp, Universidad Diego Portales}

En el Laboratorio de Innovación LincUdp se han desarrollado una serie de materiales biobasados en residuos y en sus aplicaciones con exitosos resultados (Grove \& De Saint Pierre, 2015). Los proyectos y productos que han desarrollado han sido iniciados en la carrera de diseño industrial como proyectos de título. Actualmente, algunos de éstos se han transformado en productos posicionados en el mercado nacional. Entre sus proyectos relevantes se cuentan:

- Biogusto, de Valentina Montenegro; es un producto hecho a base de cascarilla de arroz, residuo de la industria arrocera, la que comúnmente se elimina sin un posterior uso. Solo en Chile se desechan más de 25.000 toneladas de cascarilla de arroz al año. Este producto se presenta como una alternativa a los insumos de un solo uso mediante el uso de materias primas naturales y biodegradables que puede competir en igualdad de condiciones con el papel y el plástico (Grove \& De Saint Pierre, 2015).

- De corchos, de Paula Corrales; es un material reconstituidocompuesto de tapones de corcho reutilizados. Entre sus propiedades se puede mencionar que es ignífugo, liviano, impermeable, antibacterial, antideslizante, comprimible y elástico, aislante térmico y acústico, dieléctrico y absorbente vibratorio. El material se produce bajo nuevas técnicas de aglomeración y matrizado, adecuados a la industria nacional. Entre los productos generados con este material se cuentan taburetes infantiles y maceteros (Grove \& De Saint Pierre, 2015).

- Demaní, de Francisca Clavijo, es una línea de mobiliario infantil manufacturado con desecho industrial de cáscara de maní. Fabricado bajo la técnica de moldeo por prensado, permite generar un material que compite con el plástico, diferenciándose por ser $100 \%$ biodegradable (Grove \& De Saint Pierre, 2015).

- Loé y Kipmoss, de Cristián Arismendi, consiste en membranas absorbentes y retenedoras de humedad, hecha de musgo sphagnum. Kipmoss es una parte del embalaje de frutos. Crea una cámara de aire entre la superficie en que se aloja la fruta y aquella donde se acu- 
mula la humedad desprendida por la condensación interior de las cajas. Loé es una línea de macetas biodegradables hechas a base de una mezcla del mismo musgo y fibra de coco. Las macetas retienen el agua del riego, concentrando la humedad en su interior por lo que las plantas no requieren de un riego constante (Grove \& De Saint Pierre, 2015).

- Pulchén, María Ignacia Salazardiseñó briquetas de carozos de durazno para sazonar y ahumar carnes y verduras a la parrilla (Grove \& De Saint Pierre, 2015).

\section{Laboratorio de Materiales Biobasados LabMat}

El Laboratorio de Materiales Biobasados de la Facultad de Arquitectura y Urbanismo (FAU) se creó con la finalidad de desarrollar investigación y docencia en temáticas de materiales reconstituidos basados en residuos de diversos orígenes ("Laboratorio de Materiales Biobasados," 2019).

Entre los proyectos desarrollados en el Laboratorio se cuentan materiales conformados con matrices plásticas, tales como madera plástico y carozo de durazno aglomerados con polipropileno. Se han desarrollado además compuestos aglomerados con adhesivo poliuretano biobasado. Con las mismas matrices plásticas se ha aglomerado madera, carozo de durazno, cáscara de nuez, conos de pino, cápsulas de eucalipto, corcho y textiles. Se ha utilizado además como aglomerante una bioespuma, que se ha utilizado con cáscaras de huevo, carozos de aceituna y paja. Se ha trabajado con adobe, con cemento madera, con PVA aglomerando cáscaras de nuez. Se ha explorado además el uso del látex como aglomerante de corteza de eucalipto, cáscara de piña, textiles de algodón y retazos de cuero. Por otra parte, se ha experimentado el uso de matrices elaboradas con materiales sin aporte de plásticos, tales como el agar agar, la kombucha y el micelio de hongo. Con estos se ha aglomerado madera, concha de ostión y de mejillones, cáscara de nuez, algodón, hojas de té y café.

Algunos de los materiales y productos más destacados generados en el Laboratorio son:

- Material biodegradable con cáscara de nuez: Parodi (2016),en su proyecto de título de la carrera de Diseño Industrial de la Universidad de Chile, diseñó un material biodegradable con cáscara de nuez y PVA. Logró un material liviano, aislante térmico, no tóxico, conformable a partir de moldes y herramientas comunes en la industria maderera.

- Cápsulas de Eucalyptus globulus: Wechsler (2013), Diseñadora Industrial, académica de la Universidad de Chile, en el marco de su tesis doctoral desarrolló materiales compuestos de cápsulas de eucalipto aglomeradas con un poliuretano biobasado y con polipropileno reciclado. De este proyecto se obtuvo un material con una resistencia a la humedad superior a sus símiles en madera y propiedades mecánicas levemente inferiores que los materiales reconstituidos en madera. Posteriormente, el compuesto de cápsulas de eucalipto con poliuretano biobasado fue aplicado en una serie de productos en el marco de un ejercicio formativo de la carrera de Diseño Industrial. Se desarrollaron objetos tales como un casco de bicicleta, una bandeja para alimentación en colegios, un sistema para la enseñanza de horticultura en colegios y un juguete didáctico. Los productos desarrollados pueden observarse en la Figura 4. 


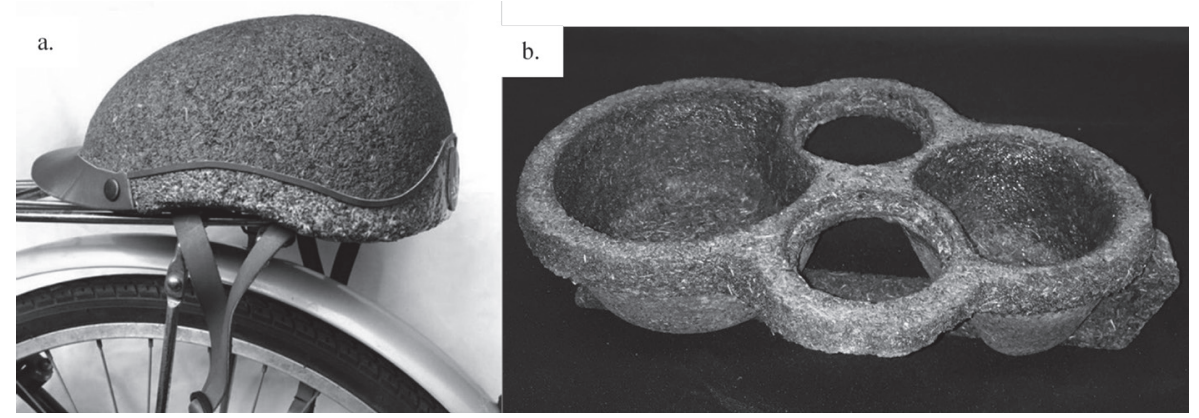

Figura 4

El eucaliptus es una especie ampliamente plantada en Brasil (más de 3.800.000 hectáreas plantadas) (Stape, 2008), Ecuador, Colombia y Chile (670.000 hectáreas plantadas) (Gysling Caselli \& Soto Aguirre, 2016). Estos árboles conforman la base de asuntos industriales tales como obtención de madera de aserrío, pulpaje, carbón y otros. Las semillas de las cápsulas de eucalipto se producen en grandes cantidades en los viveros de generación de semillas. Después que las semillas se extraen, las cápsulas se utilizan principalmente como mantillo y combustible.

Todas estas experiencias, que finalmente han ayudado a la generación de tecnología propia, han traspasado el ámbito universitario y ha comenzado a ser influyentes en distintas comunidades con consciencia medioambiental y social. En razón de ello, se ha consolidado una tendencia creciente de Diseñadores latinoamericanos dispuestos a colaborar con el desarrollo de nuevos materiales. Esto incluye la creación de nuevos materiales compuestos para los productos que ellos desarrollen, nuevas aplicaciones para materiales ya existentes o modificar materiales para obtener las propiedades deseadas. Por ejemplo, Emiliano Godoy, en México (Godoylab, 2008) Diseñador Industrial y de Mobiliario de la Universidad Iberoamericana, Pratt Institute y el Danish Design School. Su mobiliario conjugatanto la sensibilidad con el material como el dominio de la artesanía. En el año 2004 diseña la Knit Chair y en 2005 el separador de ambientes Piasa, este último fabricado con madera contrachapada certificada FSC, cuerda de algodón, y cera; no incluye clavos, y todo el producto se biodegrada al final de su vida útil (Proctor, 2009).

\subsection{La homologación de materiales biobasados como materiales industriales}

Si bien estos nuevos materiales biobasados en residuos, que pueden ser replicados fácilmente, presentan una gran oportunidad de intervención en favor del medioambiente, muchos de ellos no han prosperado por variadas razones. Una de las grandes dificultades que presentan los materiales biobasados es la sustentabilidad económica; es más, muchas experiencias no han pasado de pruebas piloto. Esto depende de varias causas, una de ellas 
se relaciona con el tamaño del mercado, debido a que la demanda no alcanza a cubrir los costos ni menos el margen de contribución esperado. Esto último, es una repetición de las circunstancias que hicieron fracasar al desarrollismo original y que es enfrentado por el nuevo desarrollismo.

Otra dificultad es la caracterización de la materia prima, que, dado su origen, presenta dificultades para garantizar su homogeneidad y asegurar variación de las propiedades del material dentro de un margen deseado, en parte por no someterse a ensayos en centros certificados, debido a que la cultura DIY es esencialmente "casera".

\section{Conclusiones}

El desarrollismo tradicional, incuba la nostalgia industrial subsidiada, que aún está presente en el imaginario tecnológico chileno pero que es distante a los desafíos que impone el mercado y que puede tener un origen en el término abrupto del desarrollismo y su reemplazo, a la fuerza, por el neoliberalismo. Esta nostalgia también afecta regularmente al Diseño chileno donde es común ver una gran cantidad de proyectos o publicaciones de rescate o revalorización patrimonial de esa época que, de cierta manera, da cuenta de una disciplina con una fuerte impronta historicista más que futurista. Sin embargo, el desarrollismo reformulado también pretende la autarquía, pero a través de la competitividad y en ese escenario, la mayor independencia del abastecimiento energético, tecnológico y de materias primas, dan sentido a las comunidades sustentables, productoras de sus propios materiales, basados en sus propios desechos, provenientes de sus propios recursos. En este posible nuevo paradigma, la competitividad productiva entre países más seculares en busca de autorrealización cambia el territorio en disputa desde el utilitarismo del material industrial hacia un territorio semántico, donde hay oportunidades para los materiales bio basados debido a su significado moral y político.

Las iniciativas de independencia tecnológica que se han desarrollado en Chile han tenido poco impacto en el sector de los materiales industriales, cuestión que afecta directamente al Diseño industrial. Una explicación posible es que, en la inmensa mayoría de los casos de materiales biobasados, los resultados experimentales no han pasado de la condición de probetas de una cierta "materialidad". Sobre estas "materialidades" se han aplicado una multiplicidad de ensayos que dan a conocer propiedades que pueden ser interesantes en determinados usos, sin embargo, la falta de homologación las relega a un segundo plano, más cercano al uso que les puede dar la artesanía que la industria. Otra implicancia no menor, relacionada con lo anterior, es que las aseguradoras no están dispuestas a asumir riesgos, como sucede en el sector de la construcción, donde todo el material debe estar certificado. Lo mismo ocurre en otras áreas vinculadas a la tecnología que resultan de riesgo y que están reguladas por marcos legales, tal como ocurre con la electricidad, combustibles, transporte, alimentos, entre otros.

A pesar de todo, existen muchos materiales biobasados que han tenido éxito, como el caso del PLA en sus diversas aplicaciones, como pueden ser las bolsas plásticas o como insumo para la impresión 3D. También existen experiencias exitosas con muchas fibras vegeta- 
les o carozos combinados con termoplásticos que dan lugar a materiales compuestos. En cualquier caso, el éxito comercial va asociado a resolver un problema específico para el cual existe un mercado interesado y que eventualmente está dispuesto a pagar más por un material bio basado. Esta disposición no se basa sólo en argumentos técnicos, sino que resultan ser más decisores de aceptación las motivaciones morales. Esto último implica una vinculación virtuosa entre la posible rentabilidad económica y la estructura valórica del mercado, donde los valores más cercanos a la secularidad y la autorrealización harán la distinción entre el éxito o fracaso económico de una iniciativa.

Visto desde fuera, puede existir cierta confusión entre aquellas comunidades que realizan voluntariado para mitigar los efectos de la polución, ya sea recolectando y/o reutilizando desechos, de aquellos que transforman la materia en un material útil para elaborar objetos, sean desde el artesanado, el Diseño o la industria. Esta necesaria distinción separa el interés personal de autorrealización, del de una actividad productiva inmersa en un sector económico y la validan como legítima en el actual contexto político y su proceso de mutación, impulsado por la base social.

Una posible razón del poco éxito de las comunidades que quieren lograr su autonomía sea el hecho de que la búsqueda de una calidad de vida mejor los ha llevado habitar en los suburbios o el campo y debido a la baja densidad poblacional no han podido sustentar económicamente el proceso. Las experiencias que se han demostrado exitosas corresponden a fenómenos urbanos, vinculados a la industria manejando economías de escala. Un material, para ser reconocido como tal y ser utilizado industrialmente debe ser replicable y sus propiedades caracterizadas. Para una correcta certificación, el desarrollo del material debe pasar de ser un proceso ensayos y pruebas, avaladas por la academia o centros de certificación, siguiendo una metodología establecida en normas nacionales o extranjeras.

Sin embargo, los materiales bio basados, abren la discusión a toda la comunidad y se constituyen por ahora en "demostradores sociales" mediados por tecnologías que,siendo menos intensivas en el uso de materias primas, incrementan el capital natural mediante la agregación del valor percibido.

\section{Notas:}

1. Lo extractivista ha cobrado protagonismo en las últimas décadas, pero ha perdido influencia debido a la globalización, que paradojalmente dio origen al neoliberalismo.

2. Al referirnos a "Recetas", lo hacemos debido a la simplificación que se hace de los procesos físico-químicos, en función de la masificación de la fórmula de código abierto.

3. Un gel extraído de algas

\section{Lista de referencias}

Aravena, J., \& Betancur, C. (15 de diciembre de 1999). Crisis y reconversión laboral del carbón. Proposiciones, 27, 1-20. Obtenido de http://www.sitiosur.cl/r.php?id=566. 
Ayres, R. U. (1989). Industrial metabolism. Technology and environment, 1989, 23-49.

Ashby, M. (2002). Ashby, M. F. (2002). Materials and Design: The Art and Science of Material Selection in Product Design. Butterworth-Heinemann.

Ashby, M., \& Johnson, K. (2009). Ashby, M. F., \& Johnson, K. (2009). Materials and Design: The Art and Science of Material Selection in Product Design (2 ed.):Elsevier Science.

Beretta, H., \& Gatani, M. (2007). Sustainable construction materials manufactured with peanut shells. In CEVE Conicet (Ed.). Cordoba, Argentina.

Berger, P., \& Luckmann, T. (2014). La construcción social de la realidad (Primera ed.). Buenos Aires: Amorrortu.

Bonsiepe, G. (2016). Del archipiélago de proyectos. Diseño indsutrial en Chile (1971-1973). La Plata: Nodal.

Bresser-Pereira, L. (2017). The new developmental theory: a synthesis. Journal of economic literature, 48-66. doi:10.1016/j.eunam.2017.01.002

Buitrago, F., \& Duque, I. (2013). La economía naranja- Una oportunidad infinita. Washington: BID.

Campos, A., \& López, M. (2017). ¿ ¿Sufre Chile el síndrome holandés? El sector de la minería del cobre en la economía chilena. Revista de administración y dirección de empresas, 1-22.

Castillo, E. (2010). Artesanos, artistas artífices. La escuela de artes aplicadas de la Universidad de Chile. Santiago: Ocholibros.

Cohen, D. (2007). Tres lecciones sobre la sociedad postindustrial. Madrid: Katz.

Conicet, C. (2007, 2013). Experimental Centre for Affordable Housing. Retrieved from www. ceve.org.ar

Consejo Nacional de la Cultura y las Artes. (2014). Mapeo de las industrias creativas en Chile. caracterización y dimensionamiento. Santiago: Consejo nacional de la cultura y las artes.

Cores Irago, I. (2018). Biobased Materials.(Diseñador de Producto). Escola d'Art I Superior de Disseny de Valencia, Valencia. Retrieved from https:/el-recetario.net/wp-content/ uploads/2018/07/TFT_Isaac_Memoria_LQ_001.pdf (DP-608-16)

Donoso, S. (2019). Investigación cualitativa en Diseño y Artes. Santiago: Ocholibros.

Donoso, S. \& Vielma, M.(2019) A propósito del centenario del Bauhaus; el dialoguismo comoposible evolución didáctica del Vorkurs. Concepción: Editorial Universidad del Biobío (En Prensa).

Eliade, M. (2013). El chamanismo y las técnicas arcaicas del éxtasis (segunda ed.). México: Fono de cultura económica.

El Palmar. (2009). Tablopan de Venezuela-An Opportunity to Manufacture Particleboard from Sugarcane Bagasse or Wood. Retrieved from http://www.elpalmar.com.ve

Fablab U.de Chile. (2019). Retrieved from http://www.fablab.uchile.cl/

FAO. (2012, 7 December 2012). FAOSTAT. Production-Crops. Retrieved from http:// faostat3.fao.org

FAO. (2017). Production quantities of peaches and nectarines by country 2014. Crops. Retrieved from http://www.fao.org/faostat/en/\#data/QC/visualize

Fernández, R., Allen, A., Burmester, M., Malvares, M., Navarro, L., Olszewski, A., \& Sagua, M. (1999). Territorio, sociedad y desarrollo sustentable. Buenos Aires: Espacio.

Florida, R. (2010). La clase creativa. Barcelona: Paidós. 
Frosch, R. A., \& Gallopoulos, N. E. (1989). Strategies for manufacturing. Scientific American, 261(3), 144-152.

Fuentes T., F. J., Silva Guzman, A., \& Richter, H. G. (1997). Coconut Stem Wood and Coir (Coconut Fibre): Potential Raw Materials for Particleboard Production. Journal of Tropical Forest Products, 3(1), 14.

Fuentes T., F. J., Silva Guzman, A., Richter, H. G., Sanjuan Duenas, R., \& Ramos Quirarte, J. (2007). Effect of production variables on bending properties, water absorption and thickness swelling of bagasse/plastic composite boards. Industrial Crops and Products, 26(1), 1-7. doi:10.1016/j.indcrop.2006.12.014

Fuentes Tavalera, F. J., Silva Guzman, A., Sanjuan Duenas, R., \& Ramos Quirarte, J. (2005, May 23-25, 2005). Properties of Composite Materials Manufactured with Sugar Cane Bagasse Particles and Recyled Plastic. Paper presented at the 8th International Conference on Woodfiber-Plastic Composites (and other fibers), Monona Terrace Community \& Convention Center, Madison, Wisconsin, USA.

Garner, A., \& Keoleian, G. A. (1995). Industrial ecology: an introduction. Ann Arbor, Michigan: National Pollution Prevention Center for Higher Education, University of Michigan.

Gilmore, J., \& Pine, J. (2011). The Experience Economy. Boston: Harvard Business Review Press.

Godoylab. (2008). GodoyLab design Work by Emiliano Godoy. Retrieved from www.godoylab.com

Graedel, T., \& Allenby, B. (1995). Industrial Ecology. 1995. Prentice Hall, Englewood Cliffs, New Jersey, 7632, 83-187.

Graham, P. (2003). Building Ecology: First principles for a sustainable built environment (1 ed.). Oxford, Great Britain: Blackwell Science (UK).

Grann, H. (1997). The industrial symbiosis at Kalundborg, Denmark. The industrial green game. Implications for environmental design and management, 117-123.

Grove, E., \& De Saint Pierre, T. (2015). Innovacion en economia circular 2015 Emprender valorizando el residuo (G. Zanetti Ed.). Santiago: Ediciones UDP.

Gysling Caselli, J., \& Soto Aguirre, D. (2016). Industria Forestal Primaria en Chile. Período 2006 -2015. Chile.

IdeaConsultora. (2012). Actualización del catastro de la agroindustria hortofrutícola chilena. Retrieved from Santiago: http://www.odepa.cl/wp-content/files_mf/1383165686Catastro_ Agroindustrial_Informe_final.pdf

iQonsulting. (2016). Agregación de valor de frutos secos, nueces y almendras. In (pp. 161). Santiago de Chile: Odepa.

Iñíguez-Covarrubias, G., Lange, S. E., \& Rowell, R. M. (2001). Utilization of byproducts from the tequila industry: part 1: agave bagasse as a raw material for animal feeding and fiberboard production. Bioresource Technology, 77(1), 25-32.

Karana, E., Pedgley, O., \& Rognoli, V. (2014). Materials experience: fundamentals of materials and design (1a. ed.. ed.). Amsterdam: Amsterdam : Elsevier.

Kistler, D., Thöni, C., \& Welzel, C. (2015). "World Values Survey Response and Behavior. World Values Research, 8(3), 101-122.

Laboratorio de Biofabricación UC. (2019). Retrieved from http://biofab.cl/category/investigacion/ 
Laboratorio de Materiales Biobasados. (2019). In A. Wechsler (Ed.), (pp. 28). Santiago: Facultad de Arquitectura y Urbanismo, Universidad de Chile.

Larraín, J. (2001). La identidad chilena. Santiago: LOM.

López, E. (2013). Gentrificación en Chile: aportes conceptuales y evidencias para una discusión necesaria. Revista de geografía Norte Grande, 31-52.

Mapeo de las industrias creativas en Chile. caracterización y dimensionamiento. (2014). Santiago: Consejo nacional de la cultura y las artes.

Materfad. (2019). Centro de materiales de la UV. Retrieved from http://uv.materfad.com/ que-es-materfad/5/materfad-valparaiso

Materiom : Home. (2019). Retrieved from https://materiom.org/

McDonough, W., \& Braungart, M. (2002). Cradle to cradle: remarking the way we make things (1st ed. Vol. 1). New York: New York : North Point Press.

Meroni, A. (2007). Creative communities. People inventing sustainable ways of living. Milano: Polidesign.

Ley N²0.920 Ley Marco para la Gestión de Residuos, la Responsabilidad Extendida del Productor y Fomento al Reciclaje., (2016).

Molina Mora, J. (2016). Desarrollo de un material compuesto basado en carozo de durazno y polipropileno para aplicaciones constructivas.(Diseñadora Industrial). Universidad de Chile, Santiago.

Navarro, J. F. (2005). Manufacture and Evaluation of High Density Plastics and Coconut Fiber based boards. (Master in Architecture). University of Colima, Colima.

Noyes, N. (2006). Sticks, stones, mud homes: inspirational living: Hardie Grant Publishing.

Objetivos de desarrollo sostenible. (2019, 8 12). Retrieved from https://www.undp.org/ content/undp/es/home/sustainable-development-goals.html

ODEPA. (2012). Industria de frutos secos, evolución destacada y amplio potencial. In (pp. 11). ODEPA: ODEPA.

Parodi Miranda, D. (2016). Desarrollo de un material compuesto biodegradable a partir de la utilización del residuo cáscara de nuez. (Diseñadora Industrial). Universidad de Chile, Santiago.

Pino, M. (2003). Nuestra cultura tecnológica, desde sus orígenes hasta fines del siglo XIX. Santiago: Editorial Universidad de Santiago.

Plagas en bodegas Promotores de enfermedades. (2014). [artículo electrónico]. HSEC Magazine.

Powazek, D. (2002). Design for community. Indianapolis: New Riders.

Programa de las Naciones Unidas para el Desarrollo. (12 de agosto de 2019). Objetivos de desarrollo sostenible. Obtenido de https://www.undp.org/content/undp/es/home/ sustainable-development-goals.htm

Proctor, R. (2009). 1000 new ecodesigns and where to find them (1 ed. Vol. 1). London: Lawrence King Publishing Ltd.

Quintero, S. L., \& Gonzalez, L. O. (2006). Uso de fibra de estopa de coco para mejorar las propiedades mecanicas del concreto. In: Universidad del Norte: Ediciones Uninorte. 
Ramírez-Calderón, G., Caulfield, D. F., Rowell, R. M., Iñíguez Covarrubias, G., \& Toriz Gonzales, G. (2005, May 23-25, 2005). Composites with Agave Fiber and Polypropylene: Mechanical and Physical Properties. Paper presentado en la 8th International Conference on Woodfiber-Plastic Composites, Madison, Wisconsin.

Robèrt, K. (1992). The Necessary Step. Ekerlids: Falun.

Rojas, M. (2006). El imaginario: civilización y cultura del siglo XXI. Buenos Aires: Prometeo. Schwab, K. (2016). La cuarta revolución industrial. Barcelona: Debate.

Stape, J. L. (2008, October). Forest plantations in Brazil: Understanding the constraints to forest productivity. Paper presented at the SOFAC Meeting, Durham NC.

Ulloa, K. (2013). Hormigón modificado con carozos de durazno. (Ingeniero constructor). Universidad de Talca, Talca, Chile.

UNEP. (2012). Global Outlook on SCP Policies: taking action together. Retrieved from Paris: http://www.unep.fr/scp/go/pdf/UNEP_Global_Outlook_SCP_ExSum.pdf

Valenzuela, A. (2011). A new agenda for blue agave landraces: food, energy and tequila. GCB Bioenergy, 3(1), 15-24.

Weber, M. (2011). La ética protestante y el espíritu del capitalismo. México: Fondo de cultura económica.

Wechsler, A. (2004). Desarrollo de Aplicaciones del Compuesto Madera Plástico. (Diseño Industrial). Universidad del Bio-Bio, Concepción, Chile.

Wechsler, A. (2013). Sustainable Particleboards: Renewable Building Materials from Agricultural and Forestry By-products. (PhD Dissertation). The University of New South Wales, Sydney.

Wechsler, A. (2018). Materiales, diseño y sustentabilidad. RChD: creación y pensamiento, 3(5). Wilson, A. (2017). Biosphere, Noosphere, Infosphere: Epistemo-Aesthetics and The Age Of Big Data. Parallax, 23(2), 202-219. doi:10.1080/13534645.2017.1299297

\begin{abstract}
The developmentalism theory proposes that the progress of countries is based on the industrialization and import substitution, against extractive development, which has been the Latin American paradigm in recent decades. The new modes of social aggregation and the DIY culture propose a circular economy for sustainable development, including biomaterials developed by the communities themselves. However, those are not necessarily industrial materials because they lack of approval, which raises deep implications for the design.

We present a state of Latin American art, about communities that produce their own materials and some cases where design has intervened.
\end{abstract}

Keywords: biomaterials - circular economy - maker culture - sustainable design - developmentalism 
Resumo: A teoria do desenvolvimento afirma que o progresso dos países se baseia na industrialização e substituição de importações, contra o desenvolvimento extrativo, que tem sido o paradigma latino-americano nas últimas décadas. Os novos modos de agregação social e cultura DIY propõem uma economia circular para o desenvolvimento sustentável, incluindo biomateriais produzidos pelas próprias comunidades. No entanto, esses não são necessariamente materiais industriais devido à falta de aprovação, uma questão que tem implicações profundas no design. Apresentamos um estado da arte latino-americana, sobre comunidades que produzem seus próprios materiais e alguns casos em que o design interveio.

Palavras chave: Biomateriais - economia circular - cultura de criadores - design sustentável - desenvolvimentismo

[Las traducciones de los abstracts fueron supervisadas por el autor de cada artículo] 